\title{
Marketing de contenidos como estrategia de enfoque promocional en los establecimientos comerciales de la parroquia Leónidas plaza, cantón Sucre
}

\author{
Marketing of contents as a promotional approach strategy in the commercial \\ establishments of the parish Leonidas plaza, district Sucre
}

\section{Marketing de contenidos en el cantón sucre}

\author{
Lilia Villacis Zambrano. ${ }^{(1)}$ \\ Jorge Muñoz Chávez. ${ }^{(2)}$ \\ Luis Mejía Ruperti. ${ }^{(3)}$
}

(1) Universidad Laica Eloy Alfaro de Manabí, Extensión Bahía de Caráquez, Ecuador. email: lilia.villacis@uleam.edu.ec

(2) Universidad Laica Eloy Alfaro de Manabí, Extensión Bahía de Caráquez, Ecuador. email: jorge.munoz@uleam.edu.ec

(3) Universidad Laica Eloy Alfaro de Manabí, Extensión Bahía de Caráquez, Ecuador. email: luis.mejia@uleam.edu.ec

Contacto: lilia.villacis@uleam.edu.ec

Recibido: 30-04-2020

Aprobado: 01-06-2020

\section{Resumen}

El presente trabajo analiza estrategias de marketing de contenidos mediante uso de redes sociales como Facebook, Instagram y WhatsApp para un público objetivo con el propósito de comunicar y divulgar el servicio que presentan establecimientos comerciales de la parroquia Leónidas Plaza del cantón Sucre. El objeto de estudio permite conocer las estrategias de marketing para verificar su aplicación, sin olvidar que el marketing de contenidos facilita atraer y retener a los clientes con la constante creación de contenidos con la intención de cambiar y mejorar el comportamiento del consumidor a su favor. Los métodos de investigación aplicados fueron el descriptivo, analítico sintético, técnica de encuesta, entrevista, y la observación, además del método Delphi. El tratamiento de la información fue realizado mediante la herramienta estadística de ciencias sociales llamada SPSS, los resultados de la herramienta permiten concluir la deficiente aplicación de estrategias de marketing digital para la atracción de clientes potenciales por lo que es necesario que se dé a conocer las nuevas estrategias que se están aplicando en la actualidad junto a la tecnología como las redes sociales.

Palabras clave: Marketing de contenidos, estrategia de viralidad, mensajes publicitarios, redes sociales, clientes potenciales

\begin{abstract}
This paper analyzes content marketing strategies through the use of social networks such as Facebook, Instagram and WhatsApp for a target audience with the purpose of communicating and disseminating the service presented by commercial establishments in the Leonidas Plaza parish of the Sucre canton. The object of study allows to know the marketing strategies to verify its application, without forgetting that content marketing facilitates attracting and retaining customers with the constant creation of content with the intention of changing and improving consumer behavior in their favor. The research methods applied were descriptive, synthetic analytical, survey technique, interview, and observation, in addition to the Delphi method. The treatment of the information was carried out by means of the statistical tool of social sciences called SPSS, the results of the tool allow to conclude the deficient application of digital marketing strategies for the attraction of potential clients so it is necessary that the new ones be made known strategies that are currently being applied alongside technology such as social networks.
\end{abstract}

Keywords: Content marketing, virality strategy, advertising messages, social networks, potential customers

\section{Introducción}


El Marketing de contenidos es una estrategia que se centra en la producción de contenidos que generan valor al cliente y que tienen únicamente un enfoque promocional y comercial. A través de los contenidos, los negocios captan la atención de los clientes, estableciendo relación y compromiso con ellos

Content Marketing Institute (2016), entidad importante en el mundo del marketing define al marketing de contenidos como "Una técnica de marketing que consiste en la creación y distribución de contenido valioso, pertinente $\mathrm{y}$ coherente para atraer y adquirir un público definido con claridad, con el objetivo de impulsar la acción rentable de los clientes" (Weareacontent, 2019).

Dentro de un emprendimiento al implementar la técnica de marketing de contenidos se estimulan los deseos de consumir mediante las imágenes y palabras claves del servicio y producto oferente hacia el cliente potencial que estimulen el deseo para el consumo. Según Joe Pullizzi fundador del Content Marketing Institute, define al marketing de contenidos como el arte de la comprensión de lo que sus clientes necesitan saber, para suministrárselo a los mismos de una manera relevante y convincente (Gnmedia, 2018).

En este sentido capturar su atención a través de un buen contenido proporciona la oportunidad de presentar llamadas a la acción para comprar o probar tu producto o servicio. Al momento de la exposición del contenido publicitario comercial, es útil su aplicación en las redes sociales que estén en tendencia de acuerdo con el momento de sociedad viral digital, para logra la comunicación y logra la captación a una grande cantidad de clientes y poder obtener subidas en las ventas del emprendimiento. Como parte del marketing de contenidos se encuentra la estrategia de viralidad como capacidad de difusión de contenidos mediante replicas y difusión masiva.

Según Steve Jurvetson (1996), el marketing viral se refiere a las personas expertas en información, personas con tendencias a recolectar datos $\mathrm{y}$ compartirlos, se caracteriza por operar en zonas en las que es difícil identificar a los consumidores. Las organizaciones, en el afán de obtener una ventaja competitiva, emplean este tipo de marketing. Hernández \& Maubert (2009), afirman que la estrategia de viralidad comienza con la recopilación de datos para la operación de contenidos que serán difundidos de manera masiva y esto será realizado con base a la información preliminar de acuerdo con las tendencias en redes de comunicación que permitirán captar futuros consumidores mediante la publicidad.
Shukla (2010), menciona que el marketing dentro de los medios digitales ofrece oportunidades que las organizaciones nunca han experimentado aun y cuando pueden realizarse estrategias mercadotécnicas simultáneas con medios tradicionales. También menciona que es importante enfatizar no tomar a los usuarios de medios digitales como tales, sino que sean vistos como potenciales clientes, ya que medios como Internet ofrecen una alta influencia manipuladora entre los usuarios involucrados, (acacia). También afirma que los medios digitales y sus usuarios representan una oportunidad para atraer clientes a un negocio nuevo, por lo tanto, es importante conocer sus necesidades y lograr persuadirlos a través de la publicidad.

El director de la revista Wired, Kevin Kelly en 1999, destaca el papel esencial que juega la tecnología en este nuevo escenario, que se ha convertido en el centro de las vidas de las personas. En sus reflexiones, Kelly sostiene que la penetración de la tecnología responde a un modelo de crecimiento orgánico: «Todos los días vemos pruebas de crecimiento biológico en sistemas tecnológicos. Esta es una de las características de la economía interconectada: la biología ha echado raíces en la tecnología y este es uno de los motivos por los que las redes lo cambian todo" (Coll \& Lluís, 2018). También aseveran que se trata de un modelo de crecimiento de los emprendimientos, la tecnología se ha convertido en el centro de los negocios para ofertar los bienes o productos, en un mercado que está en constante interacción entre usuarios y conocer también el movimiento de la competencia. El marketing de contenidos tiene la posibilidad de actualizarse cada día con las nuevas tendencias en tecnología, y la estrategia de viralidad que se presenta en las plataformas virtuales principalmente en las redes sociales son la mejor vía para presentar un negocio con su publicidad, enfatizando en el contenido de la razón de su negocio y así llegar a atraer a los posibles clientes a adquirir los mismos.

La presente investigación, tiene por objetivo conocer las estrategias de marketing que permiten verificar su aplicación, sin olvidar que el marketing de contenidos facilita atraer y retener a los clientes con la constante creación de contenidos con la intención de cambiar y mejorar el comportamiento del consumidor a su favor.

\section{Materiales y métodos}

La metodología, hace referencia al conjunto de procedimientos racionales utilizados para alcanzar el objetivo que rige una investigación, una exposición doctrinal o tareas que requieran habilidades, conocimientos o cuidados específicos. 
Se utilizó investigación descriptiva que se encargó de puntualizar las características de la población estudiada. Esta metodología se centró más en el "qué", en lugar del "por qué" del sujeto de investigación.

$\mathrm{Su}$ objetivo fue describir la naturaleza de un segmento demográfico, sin centrarse en las razones por las que se produce un determinado fenómeno.

Aplicando la metodología descriptiva se realizó encuestas para determinar los resultados de preferencia y gusto de los consumidores

La metodología cuantitativa es una de las dos metodologías de investigación que tradicionalmente se han utilizado en las ciencias empíricas. Se centra en los aspectos observables susceptibles de cuantificación, y utiliza la estadística para el análisis de los datos. Se contrapone a la metodología cualitativa o interpretativa. Este tipo de metodología es característico de un planteamiento científico positivista.

Se aplicó la metodología cuantitativa donde se utilizaron datos estadísticos y numéricos.

La investigación o metodología cualitativa es el tipo de método de investigación de base lingüístico- semiótica usada principalmente en ciencias sociales. Se suele considerar técnicas cualitativas todas aquellas distintas a la encuesta y al experimento.

Se aplicó la metodología de cualitativa para realizar analizas dependiendo de los datos obtenido.

Es un método de estructuración de un proceso de comunicación grupal que es efectivo a la hora de permitir a un grupo de individuos, como un todo,

utilización sistemática de un juicio intuitivo emitido por un grupo de expertos.

tratar un problema complejo. La capacidad de predicción del método Delphi se basa en la
La calidad de los resultados depende, sobre todo, del cuidado que se ponga en la elaboración del cuestionario y en la elección de los expertos consultados.

La técnica consiste en observar atentamente el fenómeno, hecho o caso, tomar información y registrarla para su posterior análisis.

Encuesta, es el procedimiento dentro de los diseños de una investigación descriptiva en la recopilación de datos mediante un cuestionario previamente diseñado, sin modificar el entorno ni el fenómeno donde se va a recoger la información ya sea para presentarlo en forma de tríptico, gráfica o tabla.

Conversación, grupos de discusión orientadas a la obtención de información cualitativa.

Observación, es un elemento fundamental de todo proceso investigativo; en ella se apoya el investigador para obtener el mayor número de datos.

La población utilizada para la muestra, fueron los clientes que visitan los comercios de la parroquia Leónidas Plaza, de los cuáles se consideró tomar una muestra de 40 personas escogidas al azar donde se obtuvieron datos suficientes para recabar la información necesaria a investigar. La información recolectada se procesó a través del software SPSS.

\section{Resultados}

En lo relativo a la pregunta acerca de la aplicación de redes sociales, dispositivos e información de interés, para conocer sobre la uso de herramientas del marketing de contenidos. Los resultados obtenidos en la investigación, se muestran en la tabla 1.

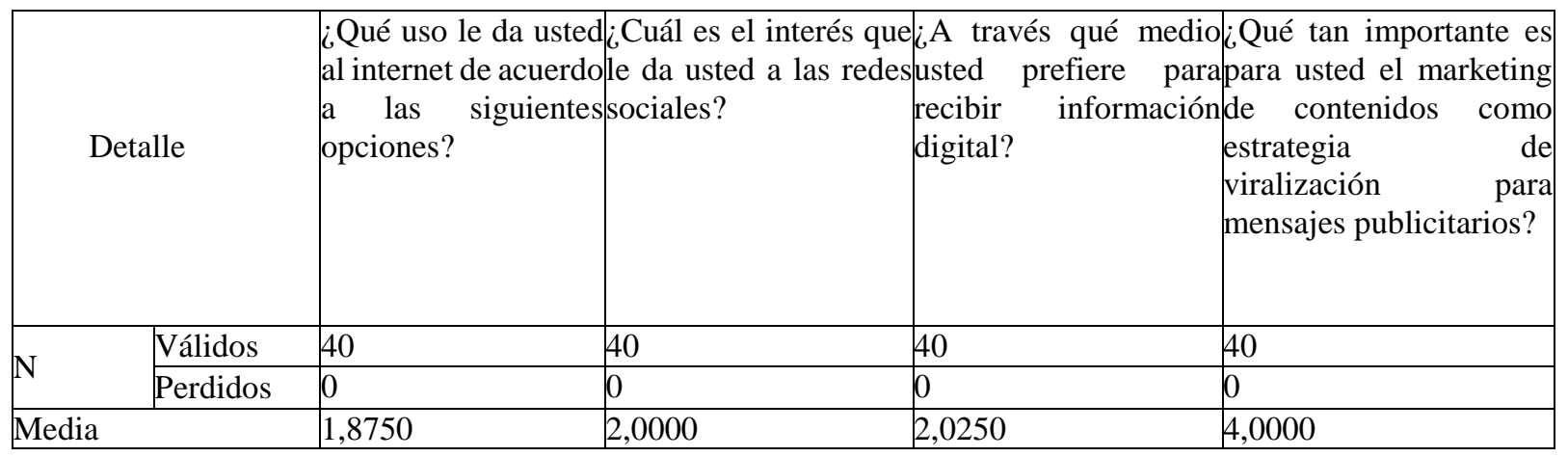




\begin{tabular}{|l|l|l|l|l|}
\hline Mediana & 2,0000 & 1,5000 & 2,0000 & 4,0000 \\
\hline Moda & 1,00 & 1,00 & 2,00 & 4,00 \\
\hline Varianza & 1,035 & 1,179 & 1,153 & 1,231 \\
\hline Suma & 75,00 & 80,00 & 81,00 & 160,00 \\
\hline
\end{tabular}

Tabla 1: Frecuencias estadísticas

En la tabla 2, se revelan los resultados de los 40 sujetos consultados, en lo relacionado al uso que se le da a la Internet, 18 sujetos respondieron que la utilizan como herramienta de trabajo, es decir el
$45 \%$; 14 personas respondieron que lo utilizan como medio de comunicación, 5 personas para investigar y finalmente 3 personas respondieron que lo utilizan para aprendizaje.

\begin{tabular}{|l|l|l|l|l|l|}
\hline \multicolumn{2}{|c|}{} & Frecuencia & Porcentaje & $\begin{array}{l}\text { Porcentaje } \\
\text { válido }\end{array}$ & $\begin{array}{l}\text { Porcentaje } \\
\text { cumulado }\end{array}$ \\
\hline \multirow{5}{*}{ Válidos } & Herramienta de Trabajo & 18 & 45,0 & 45,0 & 45,0 \\
\cline { 2 - 7 } & Comunicación & 14 & 35,0 & 35,0 & 80,0 \\
\cline { 2 - 7 } & Aprendizaje & 3 & 7,5 & 7,5 & 87,5 \\
\cline { 2 - 7 } & Investigación & 5 & 12,5 & 12,5 & 100,0 \\
\cline { 2 - 7 } & Total & 40 & 100,0 & 100,0 & \\
\hline Perdidos & Sistema & 0 & 10,0 & & \\
\hline Total & & 40 & & & \\
\hline
\end{tabular}

Tabla 2. ¿Qué uso le da usted al internet de acuerdo con las siguientes opciones? Interés en las redes sociales.

En la tabla 3, se observa que de los 40 individuos consultados, 20 respondieron que su interés sobre el uso de las redes sociales se dirige a la utilización del chat y llamadas, 14 personas respondieron que mediante estas herramientas pueden mantenerse

\begin{tabular}{|c|c|c|c|c|c|}
\hline & & Frecuencia & Porcentaje & Porcentaje válido & $\begin{array}{l}\text { Porcentaje } \\
\text { acumulado }\end{array}$ \\
\hline \multirow{5}{*}{ Válidos } & Chat, Llamadas & 20 & 50,0 & 50,0 & 50,0 \\
\hline & Conocer nuevas personas & 3 & 7,5 & 7,5 & 57,5 \\
\hline & Informarse & 14 & 35,0 & 35,0 & 92,5 \\
\hline & Publicar un producto & 3 & 7,5 & 7,5 & 100,0 \\
\hline & Total & 40 & 100,0 & 100,0 & \\
\hline Perdidos & Sistema & 0 & 0,0 & & \\
\hline Total & & 40 & 100,0 & & \\
\hline
\end{tabular}

Tabla 3: ¿Cuál es el interés que le da usted a las redes sociales?

En lo concerniente a los medios para recibir información digital, en la tabla 4, 16 personas respondieron que las redes sociales es el medio preferido para recibir información digital, 15 opinaron que es el correo electrónico, mientras que 7 sujetos usan las páginas web y dos los mensajes de textos. informados, 3 personas declararon que mediante este mecanismo pueden conocer nuevas personas y finalmente 3 personas opinaron que les sirve para publicar y dar a conocer sus productos.

\begin{tabular}{|l|l|l|l|l|l|}
\hline \multicolumn{2}{|c|}{} & Frecuencia & Porcentaje & Porcentaje válido & $\begin{array}{l}\text { Porcentaje } \\
\text { acumulado }\end{array}$ \\
\hline \multirow{4}{*}{ Válidos } & Correo Electrónico & 15 & 37,5 & 37,5 & 37,5 \\
\cline { 2 - 6 } & Redes Sociales & 16 & 40,0 & 40,0 & 77,5 \\
\cline { 2 - 6 } & Mensaje de texto & 2 & 5,0 & 5,0 & 82,5 \\
\cline { 2 - 6 } & Página Web & 7 & 17,5 & 17,5 & 100,0 \\
\cline { 2 - 7 } & Total & 40 & 100,0 & 100,0 & \\
\hline
\end{tabular}




\begin{tabular}{|l|l|l|l|l|l|}
\hline Perdidos & Sistema & 0 & 0,0 & & \\
\hline Total & 40 & 100,0 & & \\
\hline
\end{tabular}

Tabla 4: ¿A través qué medio usted prefiere para recibir información digital?

En lo referente al marketing de contenidos, en la tabla 5, muestra los resultados sobre la importancia del marketing de contenidos como estrategia de viralización para mensajes publicitarios, en este contexto, el $40 \%$ la población consultada lo considera importante, el $37.5 \%$ muy importante, para el $15 \%$ de la población es indiferente y para el $7.5 \%$ nada importante.

\begin{tabular}{|l|l|l|l|l|l|}
\hline \multicolumn{2}{|c|}{} & Frecuencia & Porcentaje & Porcentaje válido & $\begin{array}{l}\text { Porcentaje } \\
\text { acumulado }\end{array}$ \\
\hline \multirow{5}{*}{ Válidos } & nada importante & 3 & 7,5 & 7,5 & 7,5 \\
\cline { 2 - 7 } & indiferente & 6 & 15,0 & 15,0 & 22,5 \\
\cline { 2 - 7 } & importante & 16 & 40,0 & 40,0 & 62,5 \\
\cline { 2 - 7 } & muy importante & 15 & 37,5 & 37,5 & 100,0 \\
\hline Potal & 40 & 0 & 100,0 & 100,0 & \\
\hline Total & Sistema & 40 & 100,0 & & \\
\hline
\end{tabular}

Tabla 5. ¿Qué tan importante es para usted el marketing de contenidos como estrategia de viralización para mensajes publicitarios?

\section{Discusión}

Esta investigación ha tenido como objetivo indagar sobre las redes digitales más utilizadas y su valor de importancia para la población de Leónidas Plaza, tal situación permitiría conocer las estrategias de marketing de contenidos para optimizar la dinámica del mercado de la población.

En esta investigación se concuerda con otros estudios realizados por Martínez \& Sánchez (2011), en la que plantean que la evolución de la publicidad se ha producido paralelamente al de las TIC. De este modo describen como la publicidad se insertó rápidamente en Internet $\mathrm{y}$, en la actualidad, lo ha hecho en las nuevas plataformas on line en auge: las redes sociales. También aseveran el cambio que ha experimentado la publicidad y como se manifiesta tanto en la narrativa como formalmente.

Es así que el Marketing de contenidos ha alcanzado una gran importancia en el mundo publicitario y es considerado como una de las mejores alternativas para difundir de una manera interactiva el contenido de un establecimiento, logrando atraer a clientes y mejorar el crecimiento; es por ello que con el desarrollo de las redes sociales en la actualidad se la considera como una importante estrategia para la viralidad de mensajes y crear interés en los receptores (Azpeitia, Ochoa, \& Valero, 2015).
Se coincide con otras investigaciones que plantean la importancia de generar un contenido valioso que nos haga ganar la confianza incondicional de nuestros potenciales clientes, al tiempo que nos diferencia de la competencia (Ramos, 2017).

\section{Conclusiones}

Los resultados de la investigación sobre la aplicación de marketing de contenidos mediante el uso de redes sociales como, Facebook, Instagram y WhatsApp, determinan que los usuarios de estas redes en la parroquia Leónidas Plaza, no disponen de información efectiva sobre la oferta de productos y servicios de los establecimientos, sin embargo caben destacar que el $40 \%$ de población ha manifestado que prefiere recibir información digital a través de redes sociales.

Tal situación ofrece importantes oportunidades a los establecimientos que adopten la aplicación de estrategias de enfoque promocional mediante el marketing de contenidos.

\section{Bibliografía}

Azpeitia, L., Ochoa, C. \& Valero, A. (2015). El Marketing Viral Como Estrategia Globalizadora: De la teoría de redes a las redes sociales virtuales. Recuperado de http://acacia.org.mx/busqueda/pdf/EL_MA RKETING_VIRAL_COMO_ESTRATEG 


\section{UA_GLOBALIZADORA_DE_LA_TEOR IA_DE_REDES_SOCIALES_VIRTUALE S.pdf}

Bilancio, G. (2008). Marketing: Las ideas, el conocimiento y la acción (1 ra ed.). México: Pearson Educación. Recuperado el 30 de Octubre de 2019

Blanco, L. (03 de Noviembre de 2014). ¿Qué es el marketing de contenidos? 14 definiciones. Obtenido de tindalos: https://tindalos.es/que-es-el-marketingcontenidos/

Coll, P., \& Lluís, J. (2018). Estrategias de publicidad y relaciones públicas en la era digital: los casos de estudio de Wallapop, Westwing y Fotocasa. Barcelona: Editorial UOC. Obtenido de https://ebookcentral.proquest.com/lib/ulea mecsp/reader.action?docID $=5758361 \&$ que ry=estrategia+viralidad

Cravens, D., \& Piercy, N. (2007). Marketing Estratégico (8va ed.). Madrid: McGraw Hill Companies. Recuperado el 30 de Octubre de 2019

Genwords. (s.f.). Definición de Content Marketing. Obtenido de Genwords: https://www.genwords.com/blog/definicio n-de-content-marketing

Giraldo, V. (19 de septiembre de 2019). rockcontent. Obtenido de rockcontent.: https://rockcontent.com

gnmedia. (2018). Claves imprescindibles del Marketing de Contenidos en 2018. Recuperado de gnmedia: http://www.gnmedia.es/gnmediaconsistemarketing-contenidos-gnmedia/

Hernandez, C., \& Maubert, C. (2009). Fundamentos de Marketing (1ra ed.). México: Pearson. Recuperado el 30 de Octubre de 2019

Martínez, E. \& Sánchez, L. (2011). Publicidad en internet: nuevas vinculaciones en las redes sociales. Revista de Comunicación Vivat Academia,14, (117),469-480. Recuperado de https://doi.org/10.15178/va.2011.117E.469 $-480$

Nava, I. (2018). Merca2.0. Recuperado de https://www.merca20.com/ wearecontent. (2019). ¿Qué es marketing de contenidos? Recuperado de https://www.wearecontent.com/marketingde-contenidos/que-es-marketing-decontenidos 